\title{
Optics Based Biosensor for Medical Diagnosis
}

\author{
Rashmi Priyadarshini ${ }^{1}$, C K Narayanappa ${ }^{2}$ and Veena. ${ }^{3}$ \\ ${ }^{1}$ Research Scholar, School of ECE, Reva University, India \\ ${ }^{2}$ Associate Professor, Department of Medical Electronics, MSRIT, India \\ Department of ECE, the Oxford College of Engineering, India \\ ${ }^{1}$ rashmi4priya@gmail.com, ${ }^{2}{ }^{2} \_k \_n a r a y a n a p p a @ m s r i t . e d u$ \\ ${ }^{3}$ veenachander@outlook.com
}

\begin{abstract}
Biosensors have played a major role in diagnosis of various diseases and are also associated with detection of micro-organisms and other biological components. There are various types of biosensors available in the field, each having benefits one over the other. This paper explains the basic theory and operational setup of SPR based biosensors which are fast in their performances and are real time implemented. These plasmonic based biosensors includes waveguide arrangements along with a Au/Ag bimetallic enhancement concept. One of the benefits of coupling of light source with surface electrons will give raise to surface Plasmon which is very efficient in recognition of biomolecules without any external biomarkers. Placing a second metal layer above the dielectric layer as well as below, metal-insulator-metal (MIM) waveguide had been developed. These structures allow extremely high model confinement of light. Using this structure biological analysis of blood components have been performed and the resultant signature graphs are obtained in terms of resonant frequency and wavelengths. These numerical simulation outcome shows the resonance dips of the structure, high resonant transmission contrast ratio and the resonance wavelength has a linear relationship with the refractive index of dielectric material therefore the aperture.. The numerical simulation results obtained from the transmission spectra are used to analyze the sensing characteristic of the structure.The sensitivity of the biosensor is also calculated.
\end{abstract}

Keywords: Plasmonics; blood components; Resonant frequency; wavelengths; sensitivity; Biosensor; SPR; Hemoglobin; Blood Plasma; Platelets

\section{Principle of Surface Plasmon Resonance}

Biosensors are analytical devices that converts biological reactions into measurable signals like an electrical signal which is proportional to analyte concentration. It consists of a bio recognition component and a bio transducer component. Optical biosensors consist of optical bio transducers that uses the photons in order to collect information about the analyte. Whereas, Biochips are miniaturized laboratories used for detecting, analysing or processing the biological elements. Biochips have become a boon to the researchers which enables them to quickly screen a huge number of biological elements for various purposes. Biosensors are more reliable and accurate as it is label free technology, less noisy system, no electrical power is required and highly stable device. Biosensors can be used in Clinical diagnosis and biomedicine, military applications , pollution control, Pharmaceutical and drug analysis, food safety, agriculture, Microbiology for bacterial and viral analysis and many more.

SPR based optical biosensor consists of the metal that has the capacity to absorb light has taken into consideration. In majority of the cases gold metal had been used as it gives Plasmonic signals for convenient combination of reflectance angle and wavelength. When an light source is incident on the gold surface it interacts with the free electron 
constellations that is present in the gold surface. These include the outer shell along with the conduction band electrons.

The energy of photons that are present in the incident light source are absorbed by the electrons where in the plasmons are formed. The behaviour of a photon and electron can be explained only if they have both particle and wave nature properties. When the photons are converted into plasmons both the energy and momentum has to be conserved. when the frequency of the incident light matches with the frequency of the electrons the condition of resonance is established The momentum of the photons and plasmons can be described by a vector function with both magnitude and direction. The relative magnitude of the components changes when the angle or wavelength of the incident light changes. However, as plasmons are confined to the plane of the gold film, for SPR it is only the vector component parallel to the surface that matters. Thus, the energy and the angle of incident light must match to create surface plasmon resonance.

\section{SPR Dependencies and Plasmons at Metal Dielectric Interface}

Surface Plasmon resonance depends mainly on the properties of the metal film that is been used, the wavelength of the incident light and the refractive index of the metal film. As in the refractive index are very much sensitive to temperature changes, the measurements must be taken in a defined temperature. The metals that are preferred for the senor design must have conduction band electrons capable of attaining resonance condition with the incident source wavelength. The metals that satisfy the condition is copper, gold, silver, aluminium, indium, and sodium. Comparing the metals indium is considered to be too expensive, and sodium is much reactive, copper and aluminium is broad in their SPR responses and silver has high oxidation state. Hence making gold a very convenient metal. Gold is highly resistant to oxidation and other contaminants in the atmosphere. The thickness of metal layer is of great importance. Considering the optimum thickness of a gold layer that is $\pm 50 \mathrm{~nm}$, if the thickness is above an optimum thickness the dip in the reflective light becomes shallow and below the optimum thickness it becomes broader.

SPP are coherent electrons oscillations or electromagnetic excitations which propagates between the interface to two materials such as metal and the dielectric, which results in the generation of evanescent filed that decays in the perpendicular directions. A structure having one flat interface having a metal with non conducting layer having the capability to support the plasmon polaritons is as shown in the Figure 3.1(a). The field that decays in both half of the space is shown in the Figure 3.1(b). Let us start our analysis by examining the properties of plasmon wave. If we consider that there is no changes in the plane of ydirection.

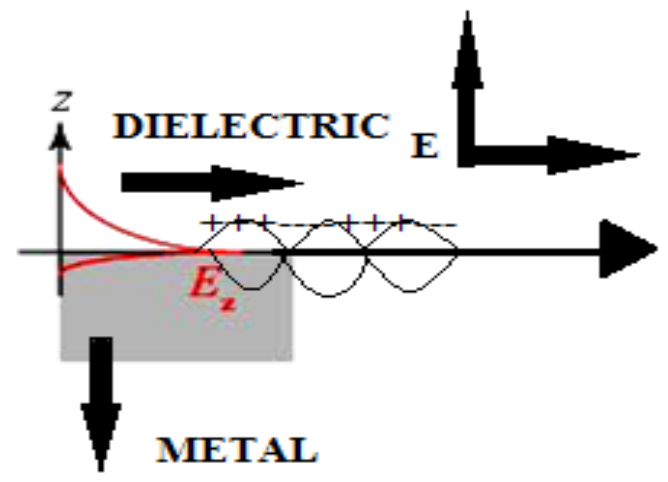

Figure 1. Schematic Illustration of the Electric Field Decay in Both Dielectric and Metal Layers 
By considering the dielectric constant or the permittivity of the material on either sides of the interface to be negligible and the time harmonic wave is $\overline{E(} \overline{r, t})=\overline{E(} \bar{r}) e-j \omega t$. The

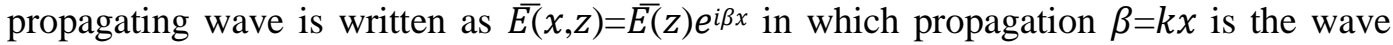
that is travelling. when we expand Maxwell's free curve equations we get the set of expressions which is given as

$$
\begin{gathered}
\partial \mathrm{Ez} \partial \mathrm{y}-\partial \mathrm{Ey} \partial \mathrm{z}=\mathrm{i} \omega \mu_{\mathrm{o}} \mathrm{Hx} \\
\partial \mathrm{Ey} \partial \mathrm{x}-\partial \mathrm{Ex} \partial \mathrm{y}=\mathrm{i} \omega \mu_{\mathrm{o}} \mathrm{Hz} \\
\partial \mathrm{Ex} \partial \mathrm{z}-\partial \mathrm{Ey} \partial \mathrm{x}=\mathrm{i} \omega \mu_{0} \mathrm{Hy} \\
\partial \mathrm{Hz} \partial \mathrm{y}-\partial \mathrm{Hy} \partial \mathrm{z}=-i \omega \varepsilon_{0} \varepsilon_{\mathrm{r}} \mathrm{Ex} \\
\partial \mathrm{Hy} \partial \mathrm{x}-\partial \mathrm{Hx} \partial \mathrm{y}=-i \omega \varepsilon_{\mathrm{o}} \varepsilon_{\mathrm{r}} \mathrm{Ez} \\
\partial \mathrm{Hx} \partial \mathrm{z}-\partial \mathrm{Hz} \partial \mathrm{x}=-i \omega \varepsilon_{0} \varepsilon_{\mathrm{r}} \mathrm{Ey}
\end{gathered}
$$

Assuming that the structure is homogeneous with respect to $\mathrm{y}$ direction so by considering $(\partial \partial y=0)$ and in the $\mathrm{x}$ direction there is a propagation of wave which given as $(\partial \partial x=i \beta)$ and The equations can be divided into independent sets

1) The magnetic modes having the $\mathrm{Hy}, \mathrm{Ex}$, and $\mathrm{Ez}$ as field components that is being non zero

2) The electric modes having $\mathrm{Hx}, \mathrm{Ey}$ and $\mathrm{Hz}$ as the field components that is being non zero

\subsection{TM Mode}

We know that the electric field will given in the form of magnetic field in that case it can written

$$
\begin{gathered}
E x=-i 1 \omega \varepsilon_{0} \varepsilon_{\mathrm{r}} \partial \mathrm{Hy} \partial \mathrm{z} \\
\mathrm{Ez}=-\beta \omega \varepsilon_{\mathrm{o}} \varepsilon_{\mathrm{r}} \mathrm{Hy}
\end{gathered}
$$

For the surface mode propagate on $\mathrm{x}$-axis and with field decay in the Z-axis, it can be written that magnetic field profile as

$$
H y=A e^{i \beta} x e^{ \pm k z} Z \text {. }
$$

For a surface mode, the real part of $\mathrm{Kz}$ is positive. The electromagnetic field of a surface mode for the upper space $(\mathrm{Z}>0)$ can be written as

$$
\begin{gathered}
\mathrm{H} y(z>0)=\mathrm{A} 1 e^{\mathrm{i} \beta \mathrm{x}} e^{-\mathrm{K}_{z}} d Z \\
\mathrm{Ex}(z>0)=\mathrm{iA} 11 \omega \varepsilon_{\mathrm{o}} \varepsilon_{r} \mathrm{~K} z d e^{\mathrm{i} \beta \mathrm{x}} e^{-\mathrm{K}_{z}} d Z \\
\mathrm{Ez}(z>0)=-\mathrm{A} 1 \beta \omega \varepsilon_{\mathrm{o}} \varepsilon_{r} e^{\mathrm{i} \beta \mathrm{x}} e^{-\mathrm{K}_{z}} d Z
\end{gathered}
$$

The electromagnetic field of a surface mode for the lower space $(\mathrm{z}<0)$ can be written as

$$
\begin{gathered}
\mathrm{H} y(z<0)=\mathrm{A} 2 e^{\mathrm{i} \beta \mathrm{x}} e^{\mathrm{K}_{z m}} Z \\
\mathrm{Ex}(z<0)=-\mathrm{i} \mathrm{A} 21 \omega \varepsilon_{0} \varepsilon_{r} \mathrm{~K} z m e^{\mathrm{i} \beta \mathrm{x}} e^{\mathrm{K}_{z m}} Z \\
\mathrm{Ez}(z<0)=-\mathrm{A} 2 \beta \omega \varepsilon_{\mathrm{o}} \varepsilon_{r} e^{\mathrm{i} \beta \mathrm{x}} e^{\mathrm{K}_{z m}} Z
\end{gathered}
$$

According to the boundary condition, the continuity of $\mathrm{Hy}$ at the interface $(\mathrm{z}=0)$ requires $\mathrm{A} 1=\mathrm{A} 2$ while continuity of Ex at the interface requires

$$
\varepsilon_{\mathrm{m}} \varepsilon_{\mathrm{d}}=\mathrm{KZ}_{\mathrm{m}} \mathrm{K} \mathrm{Z}_{\mathrm{d}}
$$

One of the condition that has to be satisfied for the generation of SPP is that the two materials should posses permittivity of opposite signs so in our case one of the materials considered to be dielectric and other to be a metal. The wave number has the following relations

$$
\begin{aligned}
\varepsilon_{\mathrm{m}} \mathrm{Ko}_{2} & =-\mathrm{KZm}_{2}+\beta_{2} \\
\varepsilon_{\mathrm{d}} \mathrm{Ko}_{2} & =-\mathrm{Kzd}_{2}+\beta_{2}
\end{aligned}
$$

We can get the expression for the propagation constant along the $\mathrm{x}$ direction with the permittivity of the two materials are

$\mathrm{E}$ across boundary is continuous: $\mathrm{E} x_{m}=\mathrm{E} x_{d} \mathrm{H}$ across boundary is continuous: $\mathrm{H} y_{m}=\mathrm{H} y_{d}$

$$
\begin{gathered}
\mathrm{K} z_{m} \varepsilon_{m} \mathrm{H} y_{m}=\mathrm{K}_{d} \varepsilon_{d} \mathrm{H} y_{d} \\
\mathrm{Kz} z_{\mathrm{m}}=-\mathrm{i} \sqrt{ } \beta_{2}-\varepsilon_{\mathrm{m}} \mathrm{k}_{2} \\
\mathrm{Kz}=-\mathrm{i} \sqrt{\beta_{2}-\varepsilon_{\mathrm{d}} \mathrm{k}_{2}} \\
-i \sqrt{ } \beta_{2}-\varepsilon_{\mathrm{m}} \mathrm{K}_{2} \varepsilon_{\mathrm{m}}=-i \sqrt{ } \beta_{2}-\varepsilon_{\mathrm{d}} \mathrm{K}_{2} \varepsilon_{\mathrm{d}}
\end{gathered}
$$


where $\mathrm{k}=\omega / \mathrm{c}$ is the wave number,

$$
\begin{gathered}
\left(\sqrt{ } \beta_{2}-\varepsilon_{\mathrm{m}} \mathrm{K}_{2}\right) \varepsilon_{\mathrm{d}}=\left(\sqrt{ } \beta_{2}-\varepsilon_{\mathrm{d}} \mathrm{K}_{2}\right) \varepsilon_{\mathrm{m}} \\
\beta_{2}=\mathrm{k}_{2}\left(\varepsilon_{\mathrm{m}} \varepsilon_{\mathrm{d}} \varepsilon_{\mathrm{m}}+\varepsilon_{\mathrm{d}}\right) \text { where } \mathrm{K}=\omega \mathrm{C} \\
\beta=\mathrm{k} \sqrt{ } \varepsilon_{\mathrm{m}} \varepsilon_{\mathrm{d}} / \varepsilon_{\mathrm{m}}+\varepsilon_{\mathrm{d}}
\end{gathered}
$$

$\lambda$ wavelength.

$\varepsilon_{d}$ and $\varepsilon_{m}=\varepsilon_{m}{ }^{\prime}+\varepsilon_{m}{ }^{\prime \prime}$ will be permittivity of dielectric and the metal layer, and $i=\sqrt{ }(-1)$. Metals like the gold, silver and aluminium shows the negative real part of permittivity $\left(\varepsilon_{m}{ }^{\prime}<0\right)$ generating the surface Plasmon.

\section{Conceptualization of Blood Components in SPR}

In this paper we apply this concept of SPR to determine the behaviour characteristics of the blood components such as Hemoglobin, blood plasma and platelets. These blood components are simulated in the sensor structure using a optical simulator tool. The difference in the signature graphs are obtained. The blood components have taken into consideration to determine the ill effects and disease caused due to changes in the content of Hemoglobin and blood plasma and platelets. Elucidating that changes (degradation) in the level of Hemoglobin can lead to anaemia, changes in blood plasma can lead to a deficiency in protein content in body, the blood clotting factors is reduced and other carbon dioxide and oxygen levels in the body is also reduced. Platelets are blood components that stops bleeding by clumping and clotting blood vessels. All the three components are explained separately in each parts of the article along with their discussion and results.

\section{Geometry of Sensor}

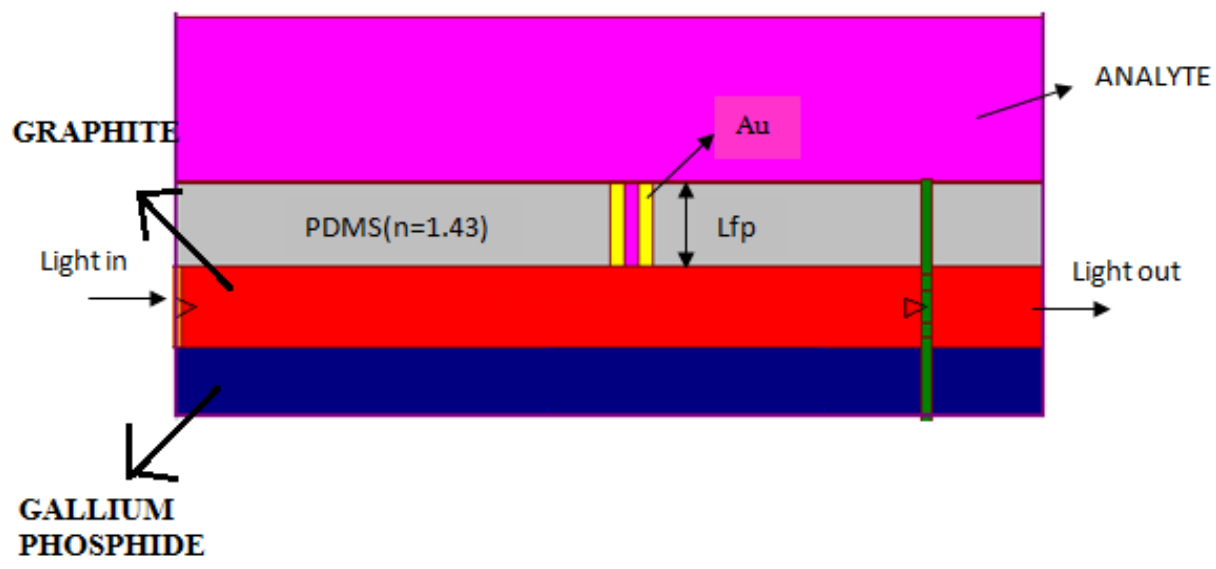

Figure 2. Structure of MIM Biosensor

The above structure is an metal insulator metal structure. These structures allow extremely high model confinement of light. The structure includes a lower substrate of a metal waveguide layer such as $\mathrm{SiO}$ and the layer placed over it is gallium phosphide which is again an optical waveguide material. When $d$ is too large for two surface Plasmons to interact, no coupling effect happens. Polydimethylsiloxane is placed as the third later on top of gallium phosphide. Polydimethylsiloxane (PDMS) belongs to a group of polymeric organoSilicon compounds that are commonly referred to as silicones. PDMS is the most widely used silicon-based organic polymer, and is particularly known for its unusual rheological (or flow) properties. PDMS is optically clear, and, in general, inert, non-toxic, and non-flammable. It is also called dimethicone and is one of several types of silicone oil (polymerized siloxane). Two parallel gold metal are placed 
parallel to each other and penpendicular to the second layer with a analyte sample placed in between the gold metal plate. This analyte is further extend as the top layer. The light source is placed at the gallium phosphide layer in order to proceed with the optical transmission and simultaneously interact with the gold metal which is the sensing medium. The graphite and gallium phosphide layer is placed below the pdms layer to order to enhance the waveguide propagation. The above concept is explained more graphically with a Figure 2 .

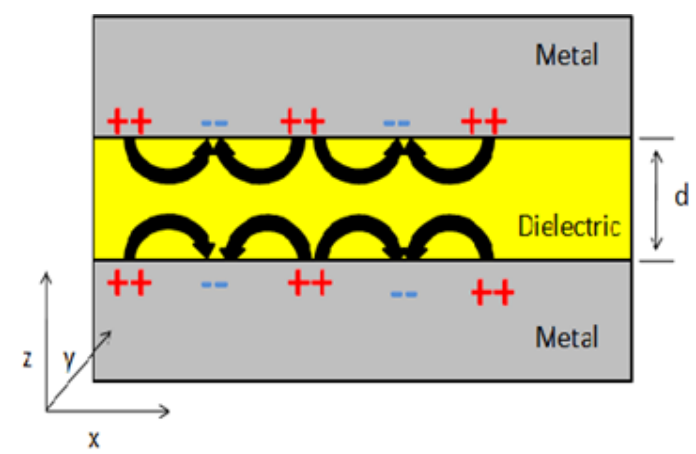

Figure 3. Surface Plasmon Polaritons in MIM Structure

As $d$ is thin enough, then interaction between two single interface surface Plasmon occurs. The smaller distance between two metal slabs, the stronger interaction it has. The effect of interaction would lead a splitting of mode in to two .one is symmetric mode, the other is anti-Symmetric one with different phases of charge oscillation distributed on the metal surface. Here the symmetry is defined as Ez with respect to the middle of dielectric layer. It's worth noting that although two kinds of modes exist in this structure, it's difficult to excite anti Symmetric mode in the real practice due to field symmetry. We have designed a tiny SPR sensor integrated on a Si waveguide based on vertical coupling into a finite thickness MIM (f-MIM) plasmonic waveguide structure acting as a FabryPerot (FP) resonator.

MIM waveguides use gap surface Plasmons that allow extremely tight mode confinement and high field enhancement and are thereby especially suitable for sensing applications. The proposed tiny SPR sensor structure is simply composed of a f-MIM plasmonic waveguide vertically coupled with a $\mathrm{Si}$ waveguide. At a resonant wavelength, for the TM-polarization (magnetic field along the y-axis), the evanescent field of the $\mathrm{Si}$ waveguide was coupled into a f-MIM plasmonic waveguide structure acting as a FP resonator. The resonant wavelength was dependent on various external parameters, such as the core (analyte) thickness, the length of the f-MIM plasmonic waveguide, and the refractive index of analyte. We can observe the transmission of the light in the MIM structure with the help of an counter map profile that is obtained using an optical simulator tool that uses FDTD principle. 

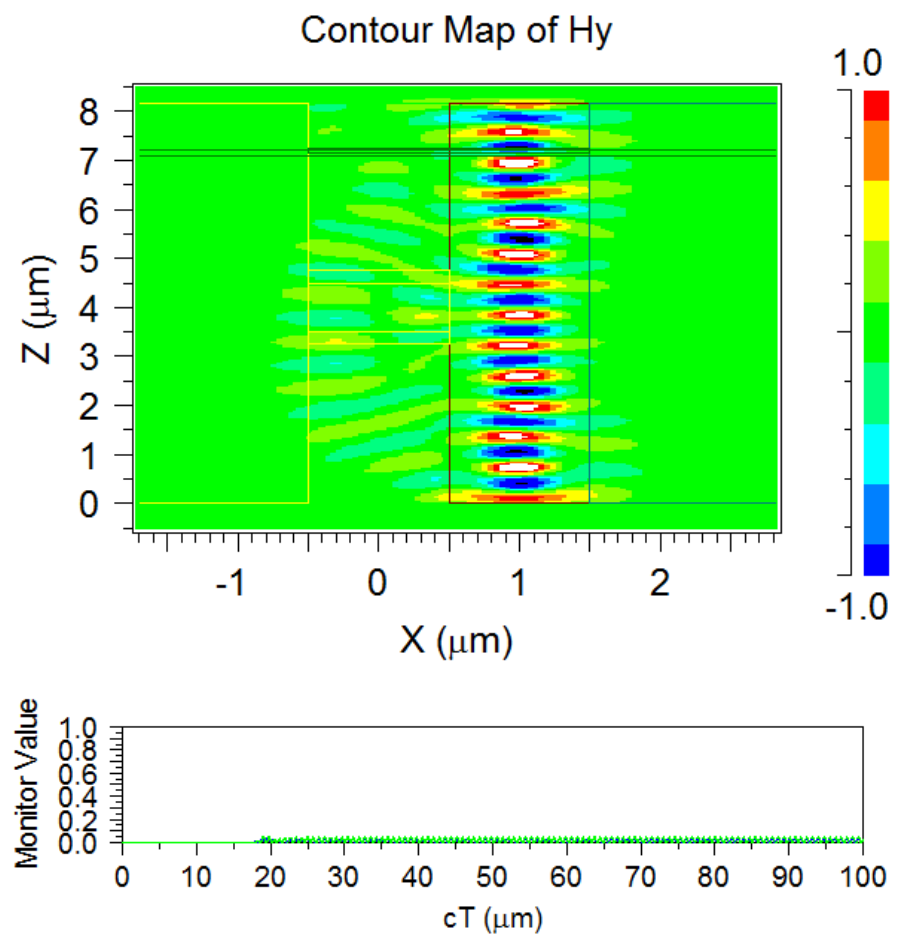

Figure 4. Transmission of Light in the Optical Waveguide Medium of Sensor

From above Figure 4 we can observe the propagation of light through the waveguide along with the interaction between the gold metal layer and the analyte that is been placed. The input source is an gausian femtosecond light source with wavelength of $1.55 \mathrm{um}$. The below table gives the parameters and the dimensions of the MIM design.

Table 1. Dimensions of Layer Design

\begin{tabular}{|l|l|l|}
\hline Layer & $\begin{array}{l}\text { Refractive index value at } \\
\text { constant } \mathbf{1 . 5 5}\end{array}$ & Thickness in $\mu \mathrm{m}$ \\
\hline \begin{tabular}{l|l} 
Dielectric layer(Au) \\
Middle
\end{tabular} & 1.4906 & $1 \mathrm{~nm}$ \\
\hline $\begin{array}{l}\text { layer(Graphite) } \\
\begin{array}{l}\text { Lower Dielectric } \\
\text { layer(GaPh) }\end{array}\end{array}$ & 3.810 & $1 \mathrm{~nm}$ \\
\hline
\end{tabular}




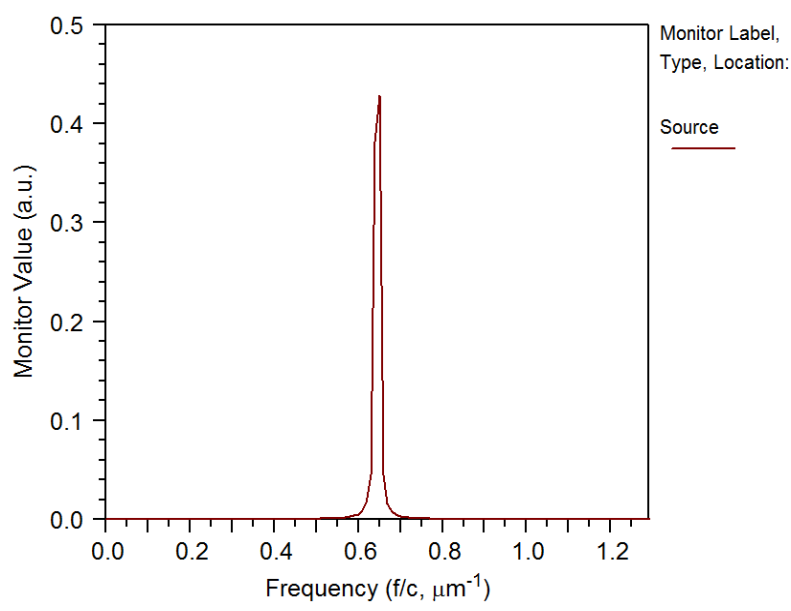

\section{Figure 5. Source Frequency Transmission Spectrum}

The above Figure 5 gives the amplitude of the source frequency with which the optical sensor was illuminated. The photons present in the source frequency are completely absorbed by the electrons present in the metal layer that interact to form the Plasmon. We can observe the output spectrum from the below graph.

Table 2. Source Frequency Tabulation
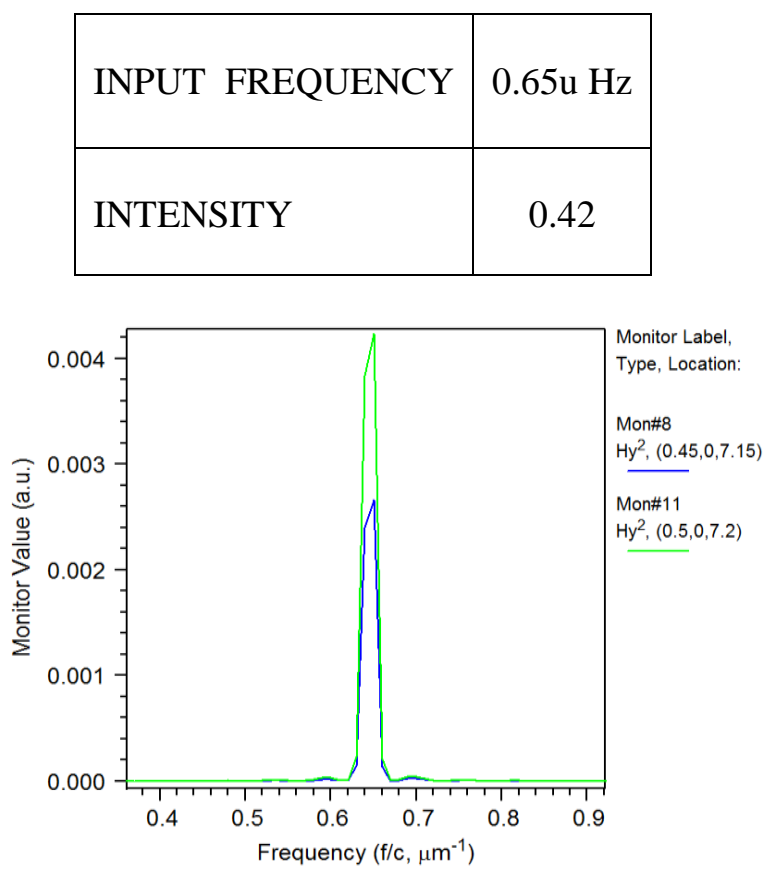

Figure 6. Output Frequency Transmission Spectrum

The above Figure 6 shows the output amplitude spectrum that is obtained after aborption of photons by the electrons. We can clearly observe that the amplitude of the output power which is very is much less than the input source amplitude of 0.425 . This input and output amplitude transmission spectrum spectrum remains the same for all the analysis of blood since the sensor taken into simulation is same for all three blood components. The resonance is observed to occur at the frequency of 0.65 
Table 3. Output Frequency Tabulation

\begin{tabular}{|c|c|}
\hline OUTPUT FREQUENCY & $0.65 \mathrm{u} \mathrm{Hz}$ \\
\hline INTENSITY & $0.004,0.0028$ \\
& \\
\hline
\end{tabular}

\section{The FullWAVE FDTD Implementation}

Two physical parameters are required in order to perform a FullWAVE simulation: the material parameters, including the relative permittivity $\varepsilon(\mathrm{r}, \omega)$ and relative permeability $\mu(r, \omega)$ as a function of space and/or frequency, and the electromagnetic field excitation. Based on these parameters, the physics dictates the electromagnetic field as a function of space and time $(\mathrm{r}, \mathrm{t})$. In order to perform a simulation, an initial launch condition $\phi L$ at time $t=0$ is needed, as well as a driving function in time. This consists of both a spatial and temporal excitation, such as

$$
\phi L(\mathbf{r}, t)=f(\mathbf{r} 0) g(t)
$$

where $f\left(\mathrm{r}_{\mathrm{o}}\right)$ is the spatial excitation at the launch plane and $g(t)$ is the temporal excitation.

\section{SPR in Analysis of Blood Components}

\subsection{Hemoglobin}

Hemoglobin is the protein molecule in red blood cells that carries oxygen from the lungs to the body's tissues and returns carbon dioxide from the tissues back to the lungs. Hemoglobin is made up of four protein molecules (globulin chains) that are connected together. Decreased levels of hemoglobin, with or without the concomitant decrease in red blood cells, can cause anemia. Iron deficiency is one cause of anemia, as it directly affects the ability to produce haem molecules, but there are several other causes of anemia. There can also be other disease profiles associated with abnormalities in hemoglobin, known generally as Hemoglobinopathies, as well as abnormalities affecting the production of haem molecules, known as porphyrias. These defected Hemoglobin cell has dielectric properties that are different from that of the normal Hemoglobin cell hence they posses different refractive index. The plasmons that are generated because of the incident light on the metal surface are highly sensitive to slight change in refractive index. These plasmons interect with the defected protein molecule and are reflected back and analysed with the help of an spectrum analyser. Hence simulating the optical sensor with known refractive index of normal Hemoglobin cell will produce a signature of graph of its own when compared to the abnormal one. With this graph we can come to an conclusion that whether the cell is affected or not. The output graph is obtained with respect to wavelength, frequency, and monitor power of the spectrum analyser. The variations of the graph are studied below. 


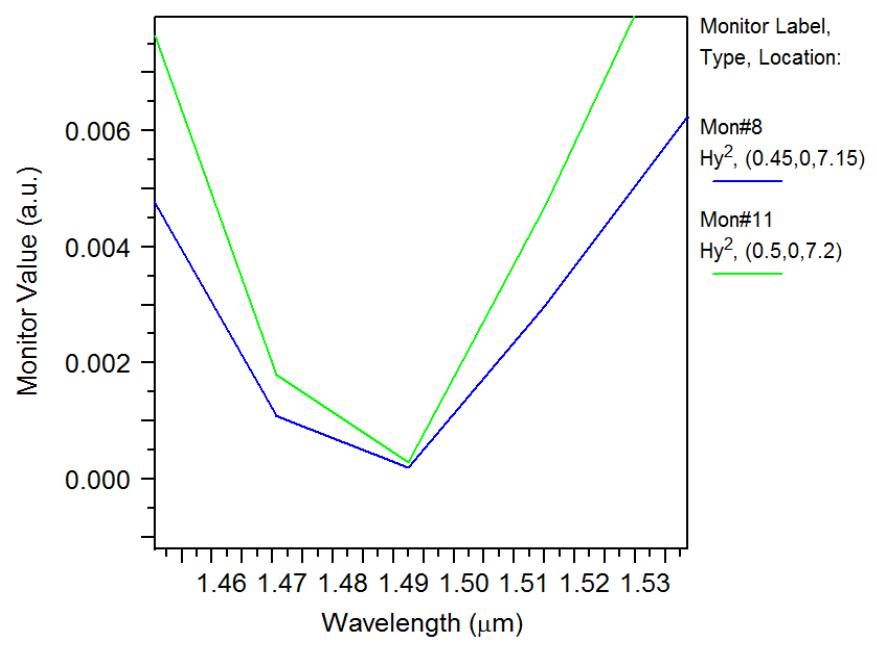

Figure 7. Wavelength Transmission Spectrum of Hemoglobin

The above Figure7 shows the wavelength transmission spectrum of Hemoglobin cell. We can observe that the amplitude intensity variation for a wavelength of $1.49 \lambda$

Table 4. Wavelength and Intensity Peaks of Hemoglobin

\begin{tabular}{|c|c|}
\hline WAVELENGTH & $1.49 \lambda$ \\
\hline INTESITY VARIATIONS & $0.00425-0.0070$ \\
\hline
\end{tabular}

\subsection{Blood Plasma}

Blood plasma is an straw coloured liquid content of cell, it includes the white blood cells, this makes the blood plasma an extracellular matrix of blood cell. Variations in this white content of the cell can lead to various interrupted diseases such as multiple myelopa and myelodysplastic syndrome. The former case is a blood cancer where a white blood cell called the plasma cell becomes a malignant one nothing but the form of tumour. These cells multiply and produce substances that damage the organs. There is no treatment for this kind of diseases but chemotherapy and stem transplant can increase the survival period. The latter consists of a family of blood cancer that effects the bone marrow. Myelodysplastic syndrome progresses very slowly but suddenly changes into a leukemia. It treatments include blood transmission and chemotherapy. As per the previous method the refractive index of blood plasma was taken and simulation was done to get the corresponding graphs. The below Figure represents the wavelength transmission of an blood plasma. 


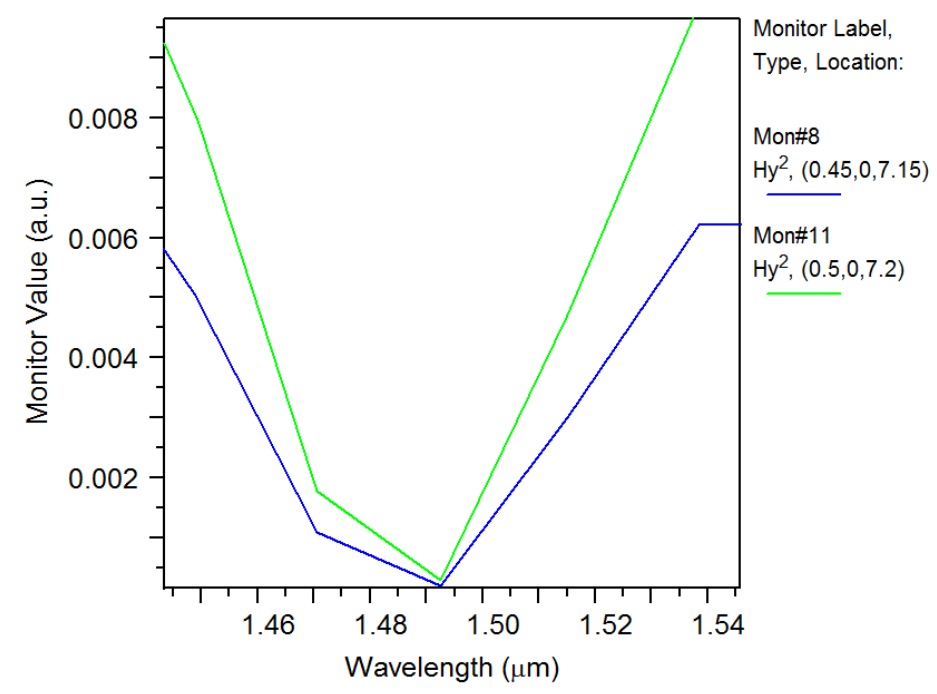

Figure 8. Wavelength Transmission Spectrum of Blood Plasma

The above Figure 8 shows the wavelength transmission spectrum of blood plasma. We can observe that the amplitude intensity variation for a wavelength of $1.49 \lambda$

Table 5. Wavelength and Intensity Peaks of Blood Plasma

\begin{tabular}{|c|c|}
\hline WAVELENGTH & $1.49 \lambda$ \\
\hline INTESITY VARIATIONS & $0.0055-0.0090$ \\
\hline
\end{tabular}

\subsection{Platelets}

Platelets are also known as thrombocytes and they are components of blood which functions to stop bleeding by clotting and clumping of blood vessels. These platelets have no nucleus in it. whenever there is wound the ligand, signal for platelets to come towards the wound.by this process more platelets will gather around the wound to give more responses as a result stops the flow of blood flowing out from the tissue. One of the disorder of platelets is thrombocytopathy. Generally normal platelets can respond to vessel wall rather than hemorrphage, which results in failure of platelets activation and thrombosiss therefore the formation of clot within an intact vessel. These variation gives rise to fibrin cot of venous thrombosis, arterial thrombosis that obstructs the flow of blood causing downstream ischemia. Considering the refractive index values of the platelets simulation were performed and graphs were obtained. The below Figure shows the wavelength transmission spectrum of an platelet cell. 


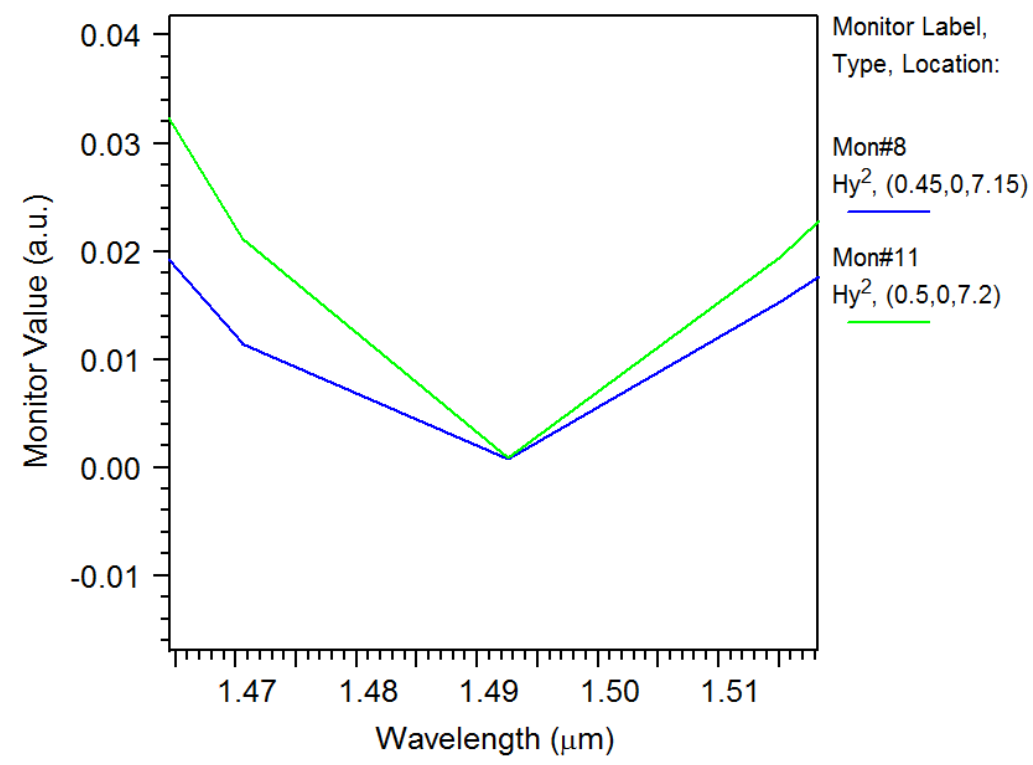

Figure 9. Wavelength Transmission Spectrum of Platelet

The above Figure 8 shows the wavelength transmission spectrum of platelet. We can observe that the amplitude intensity variation for a wavelength of $1.49 \lambda$

Table 6. Wavelength and Intensity Peaks Platelets

\begin{tabular}{|c|c|}
\hline WAVELENGTH & $1.49 \lambda$ \\
\hline INTESITY VARIATIONS & $0.0190-0.0310$ \\
\hline
\end{tabular}

All the wavelength graphs are combined to show the differences in peak and amplitude shifts of the different blood components, in order to understand the variation graphs of each blood component.

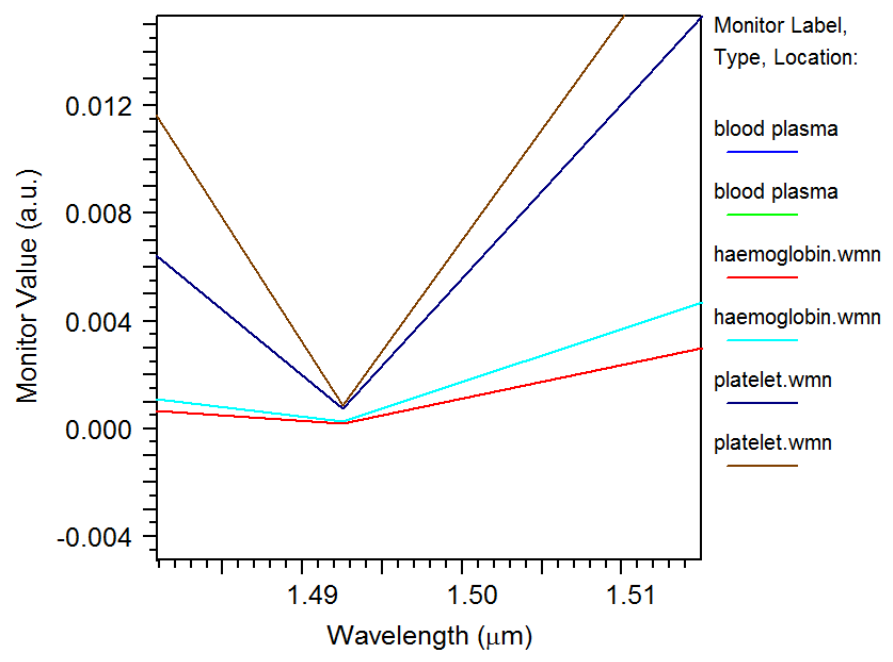

Figure 10. Wavelength Transmission Spectrum of Blood Components

From the above Figure 10 we can observe that the peak amplitude intensity for each blood components varies significantly. Hemoglobin with the lowest intensity range, platelets and blood plasma vary with shifts such as 0.0065-0.0115. 
Table 7. Wavelength and Intensity Peaks for Blood Components

\begin{tabular}{|c|c|}
\hline WAVELENGTH & $1.49 \lambda$ \\
\hline INTENSITY OF HEMOGLOBIN & 0.001 \\
\hline INTENSITY OF PLATELET & 0.0065 \\
\hline INTENSITY OF BLOOD PLASMA & 0.0115 \\
\hline
\end{tabular}

\section{Results and Discussion}

The analysis above is carried out by varying refractive index which is resulted in the proper functioning of the device by producing the shift in the wavelength thus making the proposed design compact and relatively easy to fabricate using optical lithography as compared to the conventional SPR sensor. Also metal which has a complex refractive index and as a result though SPR waveguide produces large confinement it suffers from propagation loss. Thus there is need to develop different plasmonic waveguide and has to identify the best waveguide design that can be used for the various application.

The sensor was designed on the basis of an MIM structure in order to obtain high confinement of light compared to other sensor. The sensor was tested in references with the refractive indexes of blood components such as Hemoglobin, blood plasma and platelets. Their corresponding characteristics were studied and their deficiencies were explained. The simulation was done using an optical simulator tool with an input source wavelength of $1.550 \mathrm{um}$. The signature graphs for all the components were obtained, the resonant frequency was also found along with the sensitivity. All the different signature graphs were merged together in order to show the differences between the graphs and to observe the wavelength shifts and amplitude differences.

\section{References}

[1] D. R. Shankaran, K. V. Gobi and N. Miura, "Recent advancements in surface plasmon resonance immunosensors for detection of small molecules of biomedical, food and environmental interest", Sens. Actuator. B, vol. 121, (2007), pp. 158-177.

[2] J, R. Son, A. Kothapalli, G. Kim, M. T. Morgan and D. Ess, "Detection of Salmonella enteritidis using a miniature optical surface plasmon resonance biosensor", J. Phys. Conf. Ser., vol. 61, (2007), pp. 10861090.

[3] S. D. Soelberg, G. Geiss, T. Chinowsky, C. B. Spinelli, R. Stevens, S. Near, P. Kauffman, S. Yee and C. E. Furlong, "A portable surface plasmon resonance sensor system for real-time monitoring of small to large analytes", J. Ind. Microbiol. Biotechnol, vol. 32, (2005), pp. 669-674.

[4] P. Miroslav, K. Michal and S. Petr, "Biosensors for biological warfare agent detection", Def. Sci. J., vol. 57, (2007), pp. 185-193.

[5] D. Bhatta, E. Stadden, I. J. G. Sparrow, G. D. Emmerson and E. Hashem, "Multi-purpose optical biosensors for real-time detection of bacteria, viruses and toxins", Sens. Actuator. B, vol. 149, (2010) (2010), pp. 233-238.

[6] B. Lillis, M. Manning, H. Berney, E. Hurley, M. M. Sheehan and A. Mathewson, "Dual polarisation interferometry characterisation of DNA immobilisation and hybridisation detection on a silanised support", Biosens. Bioelectron., vol. 21, (2006), pp. 1459-1467.

[7] M. A. Cooper, "Optical biosensors in drug discovery", Nat. Rev. Drug Discov., vol. 1, (2002), pp. 515528.

[8] R. Karlsson, "SPR for molecular interaction analysis: A review of emerging application areas", J. Mol. Recognit., vol. 17, (2004), pp. 151-161.

[9] C. Boozer, G. Kim, S. Cong, H. Guan and T. Londergan, "Looking towards label-free biomolecular interaction analysis in a high-throughput format: A review of new surface plasmon resonance technologies", Curr. Opin. Biotechnol., vol. 17, (2006), pp. 400-405.

[10] J. Wang, "Electrochemical glucose biosensors", Chem. Rev., vol. 108, (2008), pp. 814-825.

[11] N. J. Ronkainen, H. B. Halsall and W. R. Heineman, "Electrochemical biosensors", Chem. Soc. Rev., vol. 39, (2010), pp. 1747-1763. 
[12] B. L. Allen, P. D. Kichambare and A. Star, "Carbon nanotube field-effect-transistor-based biosensors", Adv. Mater., vol. 19, (2007), pp. 1439-1451.

[13] Z. Liu, S. Tabakman, K. Welsher and H. Dai, "Carbon nanotubes in biology and medicine: In vitro and in vivo detection, imaging and drug delivery", Nano Res., vol. 2, (2009), pp. 85-120.

[14] X. Fan, I. M. White, S. I. Shopova, H. Zhu, J. Suter and Y. Sun, "Sensitive optical biosensors for unlabeled targets", A review. Anal. Chim. Acta, vol. 620, (2008), pp. 8-26.

[15] A. Otto, "Excitation of nonradiative surface plasma waves in silver by the method of frustrated total reflection", Z. Phys. A Hadrons Nucl., vol. 216, (1968), pp. 398-410.

[16] V. Owen, "Real-time optical immunosensors-A commercial reality. Biosens", Bioelectron, vol. 12, (1997).

[17] L. Novotny and B. Hecht, "Principles of Nano-Optics", Cambridge University Press: Cambridge, UK, (2006), pp. 378-393.

[18] J. Homola, "Present and future of surface plasmon resonance biosensors", Anal. Bioanal. Chem., vol. 377, (2003), pp. 528-539.

[19] E. Kretschmann, "The determination of the optical constants of metals by excitation of surface plasmons", Z. Phys., vol. 241, (1971), pp. 313-324.

[20] R. D. Harris and J. S. Wilkinson, "Waveguide surface plasmon resonance sensors", Sens. Actuator. B, vol. 29, (1995), pp. 261-267.

\section{Authors}

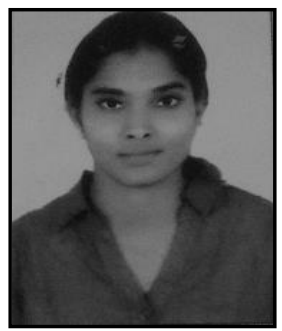

C. Veena, pursuing her M. tech under Visvesvaraya Technological University India, in Department of electronics and communication Engineering, The Oxford College of Engineering, Bangalore. Her area of research is optical biosenors, optical storage devices in nanoplatform and photonics. 
International Journal of Multimedia and Ubiquitous Engineering Vol.12, No.8 (2017) 